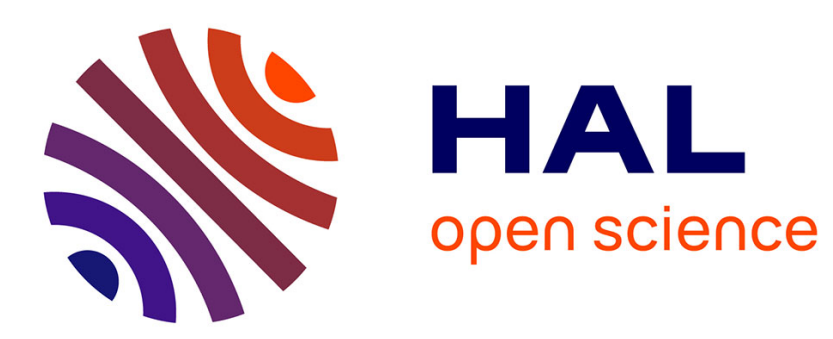

\title{
Rôle des cellules dendritiques de la muqueuse digestive dans la tolérance orale
}

\author{
B. Evrard
}

\section{To cite this version:}

B. Evrard. Rôle des cellules dendritiques de la muqueuse digestive dans la tolérance orale. Revue francaise d'allergologie, 2017, 57 (3), pp.123-124. 10.1016/j.reval.2017.02.014 . hal-01540986

\section{HAL Id: hal-01540986 \\ https://hal.science/hal-01540986}

Submitted on 16 Jun 2017

HAL is a multi-disciplinary open access archive for the deposit and dissemination of scientific research documents, whether they are published or not. The documents may come from teaching and research institutions in France or abroad, or from public or private research centers.
L'archive ouverte pluridisciplinaire HAL, est destinée au dépôt et à la diffusion de documents scientifiques de niveau recherche, publiés ou non, émanant des établissements d'enseignement et de recherche français ou étrangers, des laboratoires publics ou privés. 


\title{
Rôle des cellules dendritiques de la muqueuse digestive dans la tolérance orale
}

\author{
The role of intestinal dendritic cells in oral tolerance \\ B. Evrard ${ }^{\mathrm{a}, \mathrm{b}, *}$ \\ ${ }^{a}$ UFR médecine, UMR 1019 UNH, ECREIN, laboratoire d'immunologie, université Clermont-Auvergne, 63000 Clermont-Ferrand, France \\ ${ }^{\mathrm{b}}$ Service d'immunologie, $3^{e}$ étage centre de biologie, CHU de Clermont-Ferrand, 58, rue Montalembert, 63003 Clermont-Ferrand cedex 1, France \\ Reçu le 6 février 2017 ; accepté le 7 février 2017 \\ Disponible sur Internet le 18 mars 2017
}

Mots clés : Cellule dendritique ; Muqueuse digestive ; Tolérance orale ; T régulateurs ; Allergènes alimentaires

Keywords: Dendritic cells; Intestinal mucosa; Oral tolerance; Regulatory T cells; Food allergens

Le système immunitaire de la muqueuse digestive, communément appelé le Gut-Associated Lymphoid Tissu (GALT), est exposé en permanence à de très nombreux antigènes étrangers provenant simultanément de l'alimentation et des micro-organismes de la flore commensale intestinale. Parallèlement à cela, l'intestin représente une des principales portes d'entrée dans l'organisme des micro-organismes pathogènes. Le GALT est donc soumis à un challenge difficile, car il doit tolérer les antigènes alimentaires ou commensaux, inoffensifs voire bénéfiques, tout en restant capable de détecter et d'éliminer les antigènes issus de micro-organismes dangereux [1]. Au sein de cette muqueuse, les cellules dendritiques, véritables sentinelles de l'immunité, jouent un rôle crucial en orchestrant les réponses immunitaires innées et adaptatives, et en gouvernant en permanence le choix entre tolérance ou immunité. Néanmoins, il apparaît de plus en plus nettement que le tube digestif constitue un environnement trop complexe pour qu'une seule population cellulaire contrôle l'ensemble des types de réponses immunitaires potentielles et soit capable de les adapter finement aux diverses natures des antigènes rencontrés. Il est donc maintenant admis que ce contexte physiologique particulier a conduit au cours de l'évolution à l'apparition de différentes populations spécialisées de phagocytes mononucléés, à la fois

\footnotetext{
* Correspondance.

Adresse e-mail : bevrard@chu-clermontferrand.fr
}

des cellules dendritiques et des macrophages [2]. Les cellules dendritiques intestinales constituent ainsi un ensemble de sous-populations hétérogènes exprimant de façon différentielle un certain nombre de marqueurs phénotypiques, dont CD103, CD11b, CD172a/SIRP $\alpha$ et CX3CR1 [3]. Ces souspopulations ont des caractéristiques fonctionnelles différentes qui commencent à être de mieux en mieux appréhendées. Certaines en particulier seraient fondamentales dans la mise en place de la tolérance orale aux antigènes alimentaires, principalement les cellules dendritiques migratrices $\mathrm{CD} 103^{+} \mathrm{CD} 11 \mathrm{~b}^{-}$ et dans une moindre mesure $\mathrm{CD} 103^{+} \mathrm{CD} 11 \mathrm{~b}^{+}[4,5]$. En effet, en condition homéostasique, après migration de la Lamina propria vers les ganglions lymphatiques mésentériques, ces cellules présentent la capacité de produire de l'acide rétinoïque grâce à une enzyme, la retinaldehyde dehydrogenase 2 (RALDH2), ainsi que du TGF- $\beta$ et expriment l'indoléamine 2,3-dioxygénase (IDO). L'ensemble de ces éléments permettrait la conversion des lymphocytes $\mathrm{T}$ naïfs en $\mathrm{T}$ régulateurs périphériques $\mathrm{CD} 4{ }^{+} \mathrm{CD} 25^{+} \mathrm{FoxP} 3$ et leur homing dans la muqueuse intestinale. Ces lymphocytes seraient ensuite eux-mêmes impliqués dans l'inhibition des réponses $\mathrm{Th} 2$ et IgE spécifiques aux antigènes alimentaires, et dans la promotion des réponses IgG4 [6]. Une perturbation du fonctionnement des cellules dendritiques pourra ainsi rompre l'équilibre délicat immunité vs tolérance, générant l'apparition d'une réponse de type Th2 et d'une hypersensibilité aux allergènes alimentaires [7]. Dès lors, cibler plus finement les rôles de chacune des sous-populations de ces cellules permettra 
à terme de mieux comprendre la perte de tolérance constatée dans les allergies alimentaires et potentiellement d'améliorer leur traitement grâce à des protocoles optimisés d'induction de tolérance par immunothérapie orale.

\section{Déclaration de liens d'intérêts}

L'auteur déclare ne pas avoir de liens d'intérêts.

\section{Références}

[1] Chinthrajah RS, Hernandez JD, Boyd SD, Galli SJ, Nadeau KC. Molecular and cellular mechanisms of food allergy and food tolerance. J Allergy Clin Immunol 2016;137(4):984-97.
[2] Sanders TJ, Yrlid U, Maloy KJ. Intestinal mononuclear phagocytes in health and disease. Microbiol Spectr 2017;5(1).

[3] Watchmaker PB, Lahl K, Lee M, Baumjohann D, Morton J, Kim SJ, et al. Comparative transcriptional and functional profiling defines conserved programs of intestinal DC differentiation in humans and mice. Nat Immunol 2014;15(1):98-108.

[4] Hinterleitner R, Jabri B. A dendritic cell subset designed for oral tolerance. Nat Immunol 2016;17(5):474-6.

[5] Esterházy D, Loschko J, London M, Jove V, Oliveira TY, Mucida D. Classical dendritic cells are required for dietary antigen-mediated peripheral regulatory $\mathrm{T}$ cell and tolerance induction. Nat Immunol 2016;17(5): 545 .

[6] Wawrzyniak M, O’Mahony L, Akdis M. Role of regulatory cells in oral tolerance. Allergy Asthma Immunol Res 2017;9(2):107-15.

[7] Yu W, Freeland DMH, Nadeau KC. Food allergy: immune mechanisms, diagnosis and immunotherapy. Nat Rev Immunol 2016;16(12):751-65. 